\title{
LA PHONÉTIQUE ET LES LANGUES AFRICAINES
}

\author{
PAR A. Lloyd JAmes
}

(Voir p. 358)

Dans l'enfance nous acquérons l'usage de notre langue maternelle par de nombreuses actions mentales et physiques. C'est d'abord l'oreille et la parole qui sont intéressées, mais bien avant que cette phase ne soit achevée interviennent le toucher et la vue. Il s'ensuit que le langage se fonde sur une série compliquée d'actes et d'associations, dans lesquels l'oreille, la parole, la main, l'œil jouent un rôle et qui est probablement l'entreprise capitale de l'esprit humain. Cette complexité nous empêche de penser clairement à n'importe quel aspect, à n'importe quel acte simple. Une parole éveille pour nous l'idée des lettres qui la représentent, mais le langage auditif (parlé) n'a rien de commun avec le langage visuel (imprimé ou écrit) sauf le lien de leur signification. Rien dans la nature ne concilie la vue et le son.

Notre langue maternelle est un sérieux obstacle à l'étude des idiomes étrangers, parce qu'elle s'est imposée dans nos années d'enfance. Notre instinctif désir sera de ne proférer tout le reste de notre vie que les sons, les rythmes, les intonations de notre langue maternelle. Nous associons toujours les sons et les lettres tels qu'ils le sont dans notre langue maternelle. Un son qui éveille chez un Français l'idée de r éveillera chez un Grec celle de $\gamma$, chez un Arabe celle de $\dot{\varepsilon}$, et chez un Espagnol celle de g. Enfants, nous étions sans doute capables de proférer toutes les paroles humaines, mais par suite de l'usage constant des sons de notre langue maternelle nous perdons bientôt toute l'habileté que nous possédions au début de notre vie.

Il nous faut d'abord mettre de côté les restrictions vicieuses que nous impose notre langue maternelle. L'intelligence d'un idiome repose probablement sur les sons, les rythmes, et l'intonation. Notre but doit donc être, en étudiant un parler africain, d'analyser chacune de ses composantes et d'en estimer la valeur.

Les impressions visuelles forment une très importante contribution pour ce qui touche les sons. En règle générale elles ne fournissent aucune indication pour le rythme ou l'intonation. En ce qui concerne l'Afrique, les impressions visuelles doivent manifester, dans la plupart des cas, beaucoup plus de traits nécessaires à la compréhension que ne font les impressions visuelles dans une langue européenne.

Sons. La première tâche qui s'impose est une analyse minutieuse des sons dont nous nous servons journellement; nous pouvons ensuite acquérir la connaissance de ceux qui ne nous sont pas familiers. Le seul moyen pour cela est de les apprendre des personnes qui se sont consacrées à l'étude des sons utilisés dans toutes les langues du monde. 
Rythme. C'est l'effet produit sur l'esprit d'un observateur par le retour régulier de certaines syllabes ou de certains sons importants. Cette importance peut être due à la longueur, au diapason, ou au timbre, c'est ce qu'on nomme 'l'accent' d'une langue, mais il faut se rendre compte que ce qui constitue cet accent dans une langue n'est pas issu de la même combinaison de longueur, diapason et timbre, que dans une autre.

Intonation. Notre habitude $\mathrm{du}$ système des tons dans notre langue maternelle nous persuade qu'elle ne renferme aucun système de ce genre. Mais lorsque nous entendons parler français avec une intonation anglaise nous avons l'impression que cela n'est pas bien. Ce qui peut être obtenu par des changements d'intonations dans une langue ne peut l'être par le même changement dans une autre langue. Suivent des exemples de modifications de tons en Yorouba et en Luganda.

\section{LA BIBLIOGRAPHIE ETHNOLOGIQUE}

\section{PAR M. HEYdRICH}

$$
\text { (Voir p. 38I) }
$$

IL n'existe guère jusqu'ici de bibliographies se rapportant aux œuvres pourtant si nombreuses ayant trait à l'ethnologie, et les tentatives faites autrefois pour les réunir ont été abandonnées. On doit mentionner, cependant, des bibliographies partielles et en première ligne celle des Américanistes publiée par le Dr. P. Rivet. Il y a peu de temps encore, l'ethnologue était obligé de consulter pour se documenter les bibliographies d'autres sciences et notamment de la géographie ; quant à celles qui ne renferment que des indications ethnologiques, elles laissent beaucoup à désirer, comme le montre la table des plus importantes parmi celles qui se rapportent à l'Afrique.

C'est pour remédier à cette lacune qu'a été fondé l'Etbnologische Anzeiger, Verlag E. Schweizerbart, Stuttgart, Allemagne. La bibliographie annuelle, classée par région, comprend les publications ethnologiques et archéologiques (ces dernières pour l'Europe seulement) qui ont paru l'année précédente; les plus importants travaux, intéressant les sciences appelées à collaborer, comme l'anthropologie, la linguistique et la géographie humaine, y sont aussi mentionnés. En dehors des cuvres purement scientifiques on y trouvera l'indication d'études pouvant intéresser l'ethnologie, comme celles émanant de missionnaires ou se rapportant aux colonies ou au folklore.

Une partie spéciale formant supplément contiendra la critique des nouveautés importantes. On attirera en particulier l'attention sur les ouvrages parus dans des langues peu courantes comme le russe, le suédois, et le japonais. Enfin, une chronique particulière contiendra des renseignements sur les explorations, les événements scientifiques, les universités, les musées, et les personnalités. Les comptes rendus paraîtront en allemand, en anglais ou en français. Tout appui, toute collaboration pour cette œuvre sera bienvenu. 